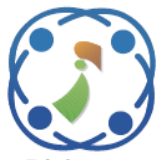

\title{
Colour Image Enhancement by Fuzzy Logic Based on Sigmoid Membership Function
}

\author{
Hazim G. Daway ${ }^{1 *} \quad$ Esraa G. Daway ${ }^{2} \quad$ Hana H. Kareem ${ }^{3}$ \\ ${ }^{I}$ Department of Physics, College of Science, Mustansiriyah University, Iraq \\ ${ }^{2}$ Department of Medical Instrumentation Techniques Engineering, Bilad AlRafidain University College, Iraq \\ ${ }^{3}$ Department of Physics, College of Science, Mustansiriyah University, Iraq \\ * Corresponding author: hazimdo@uomustansiriyah.edu.iq
}

\begin{abstract}
Colour image enhancement plays an important role in image processing, computer vision and pattern recognition. Fuzzy logic techniques one of the methods used for digital image enhancement. In this study, Fuzzy Logic Based-on Sigmoid Membership Function (FLBSMF) was developed to improve lightness and contrast in coloured images. The FLBSMF algorithm was applied to the lightness component by using only the YIQ colour space, and the colour compounds were unchanged. The suggested algorithm was compared with other algorithms, such as fuzzy logic enhancement using membership function modification based on square operation, fuzzy logic based on histogram, histogram equalisation and multiscale retinex with colour restoration by using different criteria. Form the result the FLBSMF succeeded in enhancing colour images and it had good average values for entropy (7.44), mean square error in saturation (2.2E-08) and hue (8.11E-07), nature image quality (2.97) and lightness order error (0.90).
\end{abstract}

Keywords: Fuzzy logic, Lightness enhancement, Poor contrast, Sigmoid membership function.

\section{Introduction}

In recent years, the use of digital images has increased due to the availability of technology and devices, which simplify image manipulation. When using conventional cameras, users manually control the amount of light that enters the lens by adjusting the lens size. In the direction of light, digital cameras only require a slight knowledge of the user and the principle of its work. It depends on converting light into electrical charges and the image into a series of zeros and ones to represent all the coloured points in the image [1]. Enhancement techniques aim to process a given image so that the result becomes more suitable for a specific application. Image processing methods can be divided into two parts. The first is image processing in the spatial domain by manipulating the pixel directly. The second is image processing in the frequency domain, including the Fourier, wavelet, discrete and cosine transforms [2]. Image enhancement has several types, including hazy or areal [3], underwater image [4, 5] and low lightness enhancement [6, 7] which requires increased contrast after improvement. It is one of the problems that users or researchers face when working on scientific images, such as dark medical X-rays, or old documents and photos of monuments. Multi Scale Retinex with Colour Restoration (MSRCR) [8], is one of the most important methods of enhancement color images in the low-frequency domain, in this method a logarithmic transformation is used to restore colors, and several enhancement methods have been developed using this algorithm, but one of the most important disadvantages of this method is halos effect. In the colour image enhancement field, the Fuzzy logic technology can be used in enhancing colour images; some methods used modulation in the fuzzy property plane of an image. The property domain is extracted from the spatial domain using fuzzifiers, which are important in creating different amounts of fuzziness in the plane. The fuzzy operator 'INT' (contrast intensification) is used as a tool for contrast enhancement $[9,10]$. The effectiveness of this algorithm is demonstrated on an image of 
handwritten English recursive script. A metric measure of quality for different enhanced outputs is indicated by the term 'index of fuzziness'. Studies on enhancing the contrast of different applications by preserving the brightness and improving the quality is a worldwide research problem that had been achieved by using different methods. Several researchers have attempted to address this problem, yet it persists as a major research concern. Tarun Mahashwari et al. suggested a fuzzy technique to improve the quality of an image. Various kinds of fuzzy image enhancement methods, such as fuzzy contrast adjustment, fuzzy image segmentation and fuzzy edge detection [11], have been proposed. Most researchers focused on histogram-based contrast enhancement techniques, such as homomorphic filtering; these techniques are used to enhance the low contrast of medical images [12].

In the literature, modified versions of the hyperbolic algorithm for contrast enhancement can be found. These modified algorithms are suitable for the enhancement of magnetic resonance images, this technique uses controlled fashion of the grey level, which is stretched on the entire image [13]. Some contrast enhancement techniques are based on fuzzy techniques, fuzzy techniques can manage an image's imperfection, which is modelled as the uncertainty in the image, fuzzy methods for contrast enhancement (e.g. classic fuzzy system) can be divided into three stages: fuzzification, modification of membership functions and defuzzification of the image as in [11]. In many studies, the automatic method for contrast enhancement can be found. An example of such method is grey level grouping $[14,15]$, in this method, histogram elements of low-contrast data are grouped into a proper number of classes in accordance with a selected measure; then, these classes are uniformly redistributed on the basis of lightness, and the previously grouped grey levels are ungrouped. But this method was only used to improve grey scale images when applied to colour images, false colour occurs, especially when the light is low. M. Hanmandlu et al. presented a fuzzy-based enhancement technique to improve coloured images using the Gaussian membership function, which fuzzifies the image in the spatial domain. A global contrast intensification operator, which comprises three different parameters, namely, fuzzifier, intensification parameter and crossover point, is also introduced, the HSV colour model is implemented, and the colour component is left unmodified. This approach provides visual improvement to underexposed images [16], but the most important disadvantages in this method are its complexity and its dependence on many parameters.
Harish Kundra et al. proposed a filtering technique to remove noise and improve the contrast of an image. To reach this goal, fuzzy logic with control-based approach was used and successfully improved contrast in coloured images [17]. Balasubramaniam et al. proposed a fuzzy inference system-based method of contrast enhancement for grey images (it is not used in colour images), the triangular fuzzy membership function was used to convert the pixel domain into the fuzzy domain. They proposed an algorithm to make fuzzy if-then rules specific to a given image depending on the available local information for a fuzzy inference system. To this end, a partial histogram was used, thus reducing computational costs during enhancement [18].

Preethi S.J et al. proposed Fuzzy-logic enhancement by using Membership Function Modification Depending on the Square-operation (MFMDS) and cube operator, this method has been used to enhance the usual and medical images. MFMDS algorithm increases the lighting in the medium lighting level and at low lightness levels is not good method due to the increased darkness [19].

G. Raju and S. Nairb suggested a [Fuzzy-logic based on Histogram (FLBH) algorithm as a fast method that used to enhance the colour image. In this method the HSV colour transform was used, where only the lighting component $(\mathrm{V})$ was treated by using two membership functions, this method has succeeded in preserving colour information Lowlight areas improved well, but in the regions with good lightness, after improvement, the lightness increased to be in it bleaching [20]. Denys Mikhov et al. introduced a Fuzzy-logic algorithm used to enhance contrast in the colour images, in the fuzzification implementation they suggested three membership functions are white pixel, grey pixel and Gamma-Correction. By analyzing their results the best method is Fuzzy-logic Based GammaCorrection (FLBGC). In spite of its good improvement at low light levels, But It increases lighting in the high-light region, which distorts the images in those regions [21].

In this study, we aim to improve colour images using fuzzy logic techniques, which use the Sigmoid function as a membership function for the $\mathrm{Y}$ component, on the basis of the YIQ colour space. Because of the specification of the Sigmoid function and processing of lighting component $(\mathrm{Y})$ only, the low and medium light regions are enhanced, while maintaining colour information (IQ). 


\section{Prorosed methodology}

In this study, a new method was proposed to improve the colour image by using Fuzzy Logic Based on Sigmoid Membership Function (FLBSMF). This technique was applied to the lightness (Y) component by using the YIQ colour space while preserving chromatic compounds (IQ). By using the $\mathrm{Pal}$ and King method, a grey image $\mathrm{X}$ with a size of $\mathrm{h} \times \mathrm{w}$ and a greyscale level $\mathrm{L}$ can be expressed in a fuzzy matrix with a size of $\mathrm{h} \times \mathrm{w}$ and is given by [22]

$$
X_{i j}=\bigcup_{i=1}^{h} \bigcup_{j=1}^{w} \frac{\mu_{i j}}{x_{i j}}
$$

This algorithm involves the following steps.

\section{A. Convert colour image from RGB to YIQ}

We can transform the RGB image to the YIQ model by using [23].

$$
M_{\text {for }}=\left[\begin{array}{ccc}
0.299 & 0.587 & 0.114 \\
0.596 & -0.270 & -0.322 \\
0.211 & -0.253 & 0.312
\end{array}\right]
$$

where $M_{\text {for }}$ is the forward transform of the RGB components. The range of values for the component $\mathrm{Y}$ is limited to [0-255] and should be normalised in $[0-1]$ as preparation for use in the fuzzy domain by:

$$
Y_{n}=Y / 255
$$

\section{B. Membership function transformed from lightness sets to fuzzy sets}

The fuzzy membership function in the proposed algorithm is used to optimise contrast. For the contrast enhancement, we used the S-shaped membership function. The function of membership in the S-shaped form is given by:

$$
\mu_{i j}=1 /\left(1+\left(\sqrt{\left.\left(1-X_{i j}\right) / X_{i j}\right)}\right)\right.
$$

This function is mathematically simple, as shown in Fig. 1. It does not depend on implied transactions, and it improves lighting and contrast in the fuzzy field, at low light levels, it increases light; at medium light levels, the light remains the same; at high light levels, it works to reduce the value of lighting.

A critical step is the adjustment of the organic function to provide optimal results when lighting and contrast are improved by using INT operator. If transformation $T_{1}$ has been defined, the membership functions of lightness image given by [24].

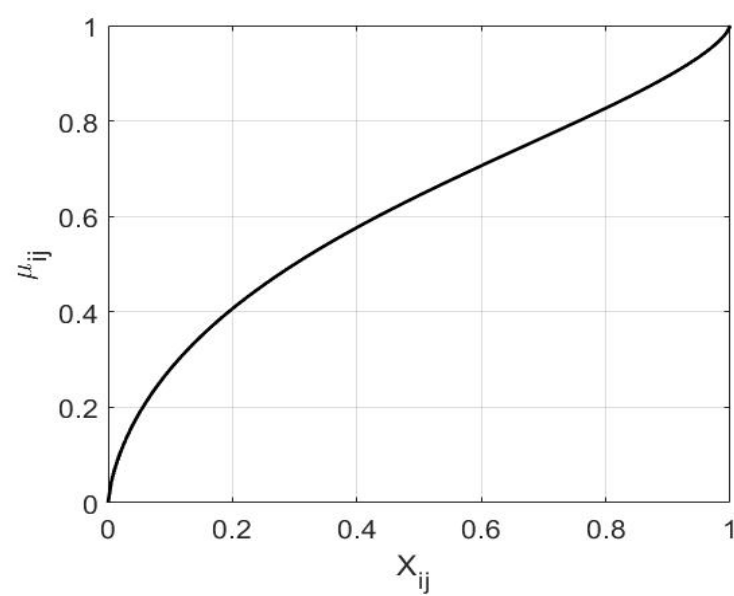

Figure. 1 Sigmoid membership function

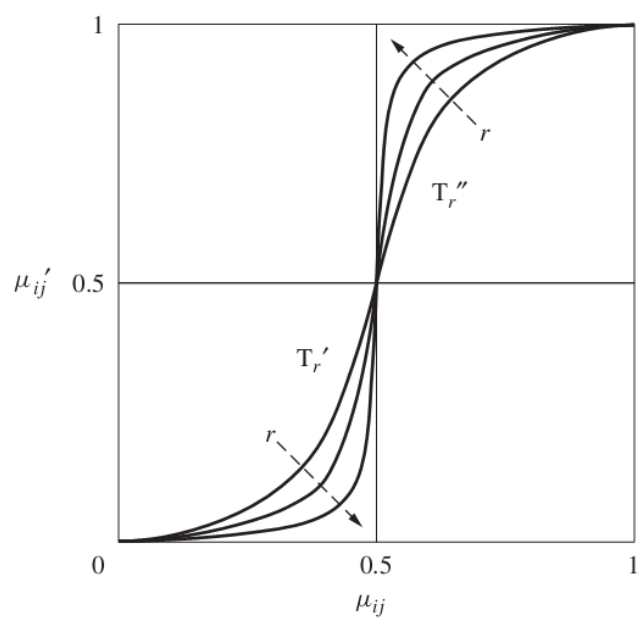

Figure. 2 Enhanced INT transformation function for contrast enhancement

$$
\begin{aligned}
& T_{1}\left(\mu_{i j}\right)=T_{1}{ }^{\prime}\left(\mu_{i j}\right) \quad \text { at } \quad 0 \leq \mu_{i j} \leq 0.5 \\
& T_{1}\left(\mu_{i j}\right)=T_{1}{ }^{\prime \prime}\left(\mu_{i j}\right) \quad \text { at } \quad 0.5 \leq \mu_{i j} \leq 1
\end{aligned}
$$

So,

$$
T_{1}\left(\mu_{i j}\right)=\left\{\begin{array}{ll}
2 \mu_{i j}^{2} & 0 \leq \mu_{i j} \leq 0.5 \\
1-2\left(1-\mu_{i j}\right)^{2} & 0.5 \leq \mu_{i j} \leq 1
\end{array}(6)\right.
$$

mostly, for $\mu_{i j}$ in image $X$ may be transformed to $\mu^{\prime}{ }_{i j}$ to enhance the $X$ in the property domain by a modification function [25].

$$
\mu_{i j}^{\prime}= \begin{cases}T_{r}\left(\mu_{i j}\right) & 0 \leq \mu_{i j} \leq 0.5 \\ T_{r}{ }^{\prime \prime}\left(\mu_{i j}\right) & 0.5 \leq \mu_{i j} \leq 1\end{cases}
$$

The modification $T_{r}$ is called as successive applications of $T_{1}$ by the recursive relation,

$$
T_{r}\left(\mu_{i j}\right)=T_{1}\left(T_{r-1}\left(\mu_{i j}\right)\right), r=1,2 \ldots
$$


recursive mapping for the membership function is illustrated in Fig. 2, as $r$ (this mean the number of successive applications of the INT operator) was increased, the slope of the curve makes steeper. As $r$ reaches to infinity, the shape becomes a crisp (binary) function. The value $r$ permit the user to use an appropriate level of improvement for domainspecific situations.

\section{Defuzzification of fuzzy set}

Adaptable fuzzy contrast defuzzification is implemented to improve contrast enhancement in terms of flexibility and effectiveness and prevent the issue of over- or under-enhancement. During defuzzification, grey-level values are retrieved from the fuzzy plane. The inverse Sigmoid Function is defined in Eq. (4). Fuzzy values between 0 and 1 are transformed into spatial domain values, i.e. between 0 and 255 , by using the inverse transform $\mathrm{Ye}=$ $\mathrm{F}^{-1} \mathrm{C}_{\mathrm{r}}\left(\mu_{\mathrm{ij}}\right)$ function by using:

$$
\mathrm{Ye}=255 /\left(1+\left(\frac{1-\mu_{i j}^{\prime}}{\mu^{\prime}{ }_{i j}}\right)^{2}\right)^{\alpha}
$$

where $F^{-1}$ is the inverse function and $\alpha$ is correction parameter is added to increasing lightness in the dim regions (we used $\alpha=0.3$ ). Lastly we converted image from the YIQ to the RGB colour space by using [23]:

$$
M_{i n v}=\left[\begin{array}{rrr}
1 & 0.956 & 0.621 \\
1 & -0.272 & 0.647 \\
1 & -1.060 & 1.703
\end{array}\right]
$$

where $M_{i n v}$ is the inverse transform. The steps of the FLSMF algorithm are as follows:

1. Read input image $c(x, y, k), k=1,2,3$

2. Transform the RGB image to YIQ space by using Eq. (2), and extract the $Y$ component.

3. Normalise the $Y$ component using Eq. (3).

4. Apply the sigmoid membership function using Eq. (4).

5. Complete the base of operations in the fuzzy domain $T_{1}\left(\mu_{i j}\right)$ using Eq. (6).

6. Perform defuzzification on the $Y$ component using Eq. (9).

7. Merge $Y e$ with the $I$ and $Q$ components to obtain $\mathrm{Y}_{\mathrm{e}} \mathrm{IQ}$.

8. Map the $\mathrm{Y}_{\mathrm{e}} \mathrm{IQ}$ colour space to $(\mathrm{RGB})_{\mathrm{e}}$ colour enhanced image using Eq. (10) to obtain the final enhanced image.

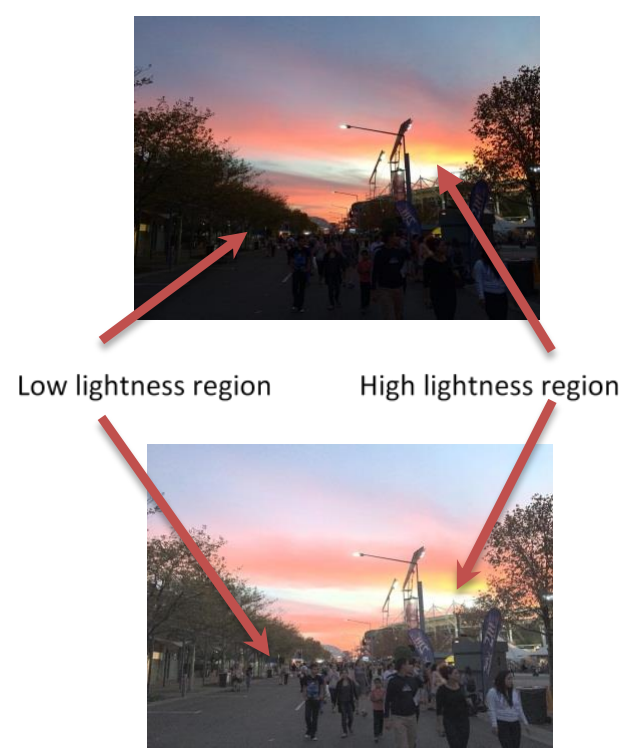

Figure. 3 Enhanced image by using FLBSMF with high and low-light regions marked

Fuzzy logic makes it possible to correct the lighting and modify the contrast using the FLSMF algorithm in the YIQ colour space while preserving the original colour characteristics of the RGB space image because of the preservation of chromatic compounds (IQ). Among the most important advantages in the proposed method, we note its success in preserving colours due to the use of colour transform after enhancement, because of the properties of the Sigmoid function and had the good enhancement on irregular lighting distribution at all levels (low and high light levels), where at the levels of lighting for the minority increase the degree of illumination after improvement and the high levels of lighting did not increase the degree of lighting and did not lead to bleaching events in these regions as in Fig. 3.

\section{Image quality assessment}

Several measures were used to measure the quality of the enhanced images.

\subsection{Lightness order error}

The Lightness Order Error (LOE) is related to the lightness relation between the original and enhanced images to measure naturalness. The lightness I (x, y) of any tested image can be expressed as the highest three colour band value [26].

$$
I(x, y)=\operatorname{Max}(c(x, y, k))
$$


where $k \in r, g, b$ for grey level $I(x, y)$ is the difference between of the original image $I$ lightness and its enhanced value $I e$. The LOE can be summarised as follows [26].

$$
\mathrm{LOE}=\frac{1}{m * n} \sum_{i=1}^{m} \sum_{j=1}^{n} D S(i, j) .
$$

The $D S$ function depending on $I e$ and $I$ and calculated by using step function, the definition of LOE tell us that the smaller value is better for the lightness.

\subsection{Natural image quality evaluator}

The natural image quality evaluator (NIQE) is a picture quality analyser that is good for evaluating variance in the original image. This analyser contrasts the picture studied to a generic standard; the lower value, the higher the level of perception. The NIQE is defined as the local mean deviation field [27].

$$
\delta=\sum \sum_{i, j} \sigma(i, j)
$$

where $\delta$ is the local sharpness, $\sigma$ is the mean filter.

\subsection{Mean squared error for hue and saturation}

This scale is used as a tool to measure how well the colour components are preserved after enhancement. The mean squared error for hue (MSEH) between the original $\mathrm{H}$ and enhanced $\mathrm{Hn}$ images is written as.

$$
M S E H=\frac{1}{M N} \sum_{x=1}^{M} \sum_{y=1}^{N}\left(\left(H_{n}(x, y)-H(x, y)\right)^{2}\right.
$$

And MSEH $\mathrm{f}$ between (the original- $\mathrm{S}$ and enhanced $\mathrm{Sn}$ ) is given by:

$$
M S E S=\frac{1}{M N} \sum_{x=1}^{M} \sum_{y=1}^{N}\left(\left(S_{n}(x, y)-S(x, y)\right)^{2}\right.
$$

The fewer the error values are in the two equations led to preserved color data.

\subsection{Entropy}

Entropy could be interpreted as image quality details corresponding to the degree of intensity. Generally, when the entropy value increases, the image retains the detailed content. Entropy is written as [1].

$$
\text { Entropy }=-\sum_{i} p(i) \log (p(i))
$$

Where $p(i)$ is the means the likelihood of gray level.

\section{Result and discussion}

In this study, we used four test images in JPEG format. All images have poor contrast intensity, making them suitable for testing the feasibility of the proposed FLSMF method. The analysis consisted of five parameters (entropy, NIQE, LOE, MSEH and MSES). These assessments were used to compare all processing methods: MFMDS [20], FLBH [21], FLBGC [22] and MSRCR [8]. Figs. 4-7 illustrate all the images enhanced by using the five methods. The subjective evaluation suggested that among the image enhancement methods used in this study, the proposed method was the best as it improved the brightness and contrast for all types of images. This enhancement was reflected in the entropy values shown in tables (1-4). The highest values of entropy (at the same time, the LOE values were small) are found in the proposed method and FLBGC algorithm. The high entropy value and small value of LOE indicate a good level of lightness enhancement, whereas the (FLBH, MFMDS and MSRCR) algorithms did not have a good lightness enhancement due small values of entropy and high values of LOE. For the two scales MSES and MSEH measure the amount of colour distortion after enhancement, the fewer the values are referred to the better the enhancement. We note the lowest values for the proposed algorithm FLBSMF, which indicates the success of the method in maintaining colour information after enhancement. While all methods (FLSM, MSRCR, FLBGC and FLBH) have a high error, this means that some distortions will occur in hue and saturation after enhancement. NIQE represents the best result for this scale when the values are small and this means that it is the best image enhancement, we can see in tables the lowest error values were found in the proposed method FLBSMF.

\section{Conclusions}

In this study, the colour images were enhanced by using the proposed FLSMF method and other methods, such as MFMDS, FLBH, HE and MSECR. We can conclude, the best results were obtained by the proposed method as it excelled in enhancing contrast and lightness in colour images. Compared 
Table 1. Quantitative criteria to compare the methods used in Fig. 2 for the girl_1 image

\begin{tabular}{|c|c|c|c|c|c|}
\hline \multirow{2}{*}{$\begin{array}{c}\text { Enhancement } \\
\text { Method }\end{array}$} & \multicolumn{5}{|c|}{ Quality Assessment } \\
\cline { 2 - 6 } & Entropy & MSE-S & MSE-H & NIQE & LOE \\
\hline FLBSMF & 7.49366 & 0.00013 & $2.52 E-06$ & 2.78672 & 0.35839 \\
\hline FLSM & 6.01177 & 0.00151 & 0.11555 & 4.40442 & 12.01914 \\
\hline MSRCR & 4.98294 & 0.00714 & 0.46839 & 7.89491 & 198.5050 \\
\hline FLBGC & 6.44576 & 0.00668 & 0.31357 & 3.61363 & 15.24573 \\
\hline FLBH & 6.19714 & 0.00466 & 0.17065 & 3.59555 & 0.56780 \\
\hline
\end{tabular}

Table 2. Quantitative criteria to compare the methods used in Fig. 3 for the minaret image

\begin{tabular}{|c|c|c|c|c|c|}
\hline \multirow{2}{*}{$\begin{array}{c}\text { Enhancement } \\
\text { Method }\end{array}$} & \multicolumn{5}{|c|}{ Quality Assessment } \\
\cline { 2 - 6 } & Entropy & MSE-S & MSE-H & NIQE & LOE \\
\hline FLBSMF & 7.37311 & $1.89 \mathrm{E}-10$ & $5.34 \mathrm{E}-09$ & 3.02259 & 1.10339 \\
\hline FLSM & 6.36706 & 0.00010 & 0.23179 & 4.11251 & 18.5535 \\
\hline MSRCR & 6.34708 & 0.00401 & 0.26662 & 3.64147 & 165.094 \\
\hline FLBGC & 7.53297 & 0.00553 & 0.12038 & 3.32722 & 12.8556 \\
\hline FLBH & 6.57252 & 0.00171 & 0.01741 & 3.41290 & 1.20760 \\
\hline
\end{tabular}

Table 3. Quantitative criteria to compare the methods used in Fig. 4 for the girl_2 image

\begin{tabular}{|c|c|c|c|c|c|}
\hline \multirow{2}{*}{$\begin{array}{c}\text { Enhancement } \\
\text { Method }\end{array}$} & \multicolumn{5}{|c|}{ Quality Assessment } \\
\cline { 2 - 6 } & Entropy & MSE-S & MSE-H & NIQE & LOE \\
\hline FLBSMF & 7.29121 & $2.2 \mathrm{E}-08$ & $2.34 \mathrm{E}-07$ & 2.40879 & 0.22048 \\
\hline FLSM & 5.90168 & 0.00038 & 0.17260 & 2.83963 & 2.26947 \\
\hline MSRCR & 6.31931 & 0.00179 & 0.39657 & 3.92561 & 173.6299 \\
\hline FLBGC & 7.43463 & 0.00135 & 0.19248 & 3.89256 & 38.25448 \\
\hline FLBH & 6.52707 & 0.00150 & 0.01730 & 2.66414 & 0.35334 \\
\hline
\end{tabular}

Table 4. Quantitative criteria to compare the methods used in Fig. 5 for the tire image

\begin{tabular}{|c|c|c|c|c|c|}
\hline Enhancement & \multicolumn{5}{|c|}{ Quality Assessment } \\
\cline { 2 - 6 } Method & Entropy & MSE-S & MSE-H & NIQE & LOE \\
\hline FLBSMF & 7.61290 & $1.33 E-06$ & $4.85 E-07$ & 3.68135 & 1.92692 \\
\hline FLSM & 6.95261 & 0.00029 & 0.42011 & 3.76361 & 35.7802 \\
\hline MSRCR & 6.63788 & 0.00067 & 0.44988 & 6.00849 & 115.6542 \\
\hline FLBGC & 7.47028 & 0.00120 & 0.10760 & 3.32058 & 1.42420 \\
\hline FLBH & 6.57807 & 0.00073 & 0.18731 & 4.09128 & 2.02037 \\
\hline
\end{tabular}

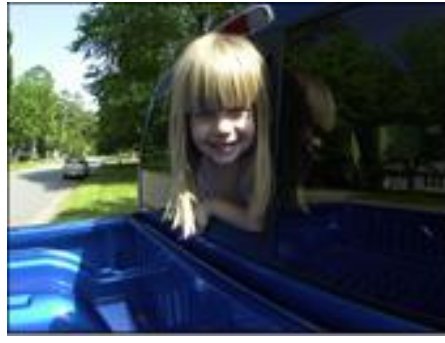

(a)

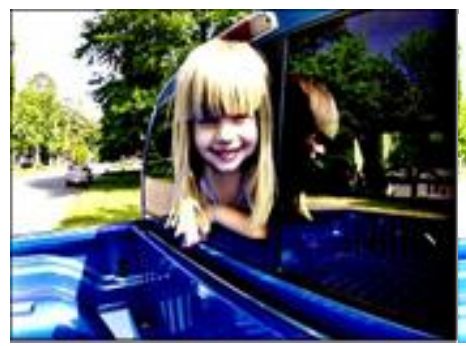

(d)

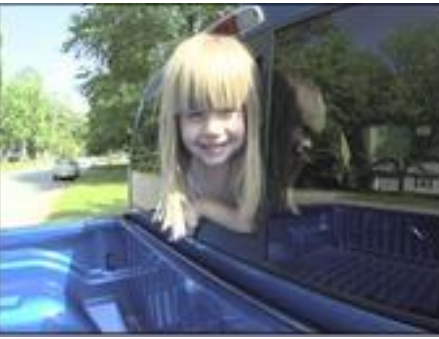

(b)

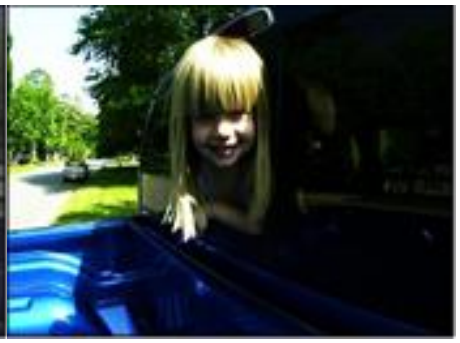

(c)

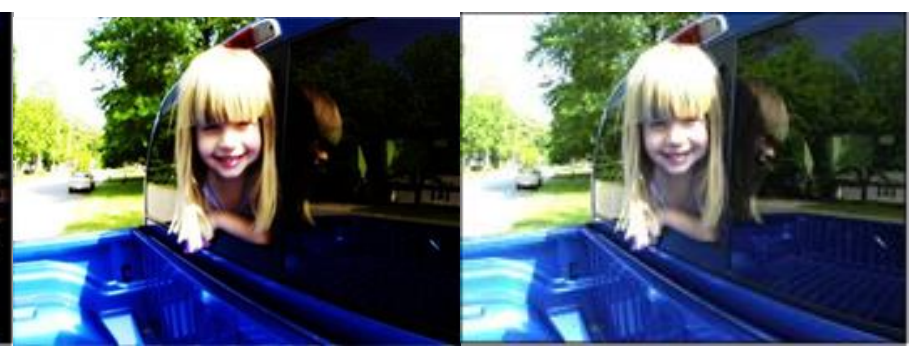

(e)

(f)

Figure. 4 Contrast-improved girl_1 images in: (a) original, (b) FLBSMF, (c) FLSM, (d) MSRCR, (e) FLBGC, and (f) FLBH 


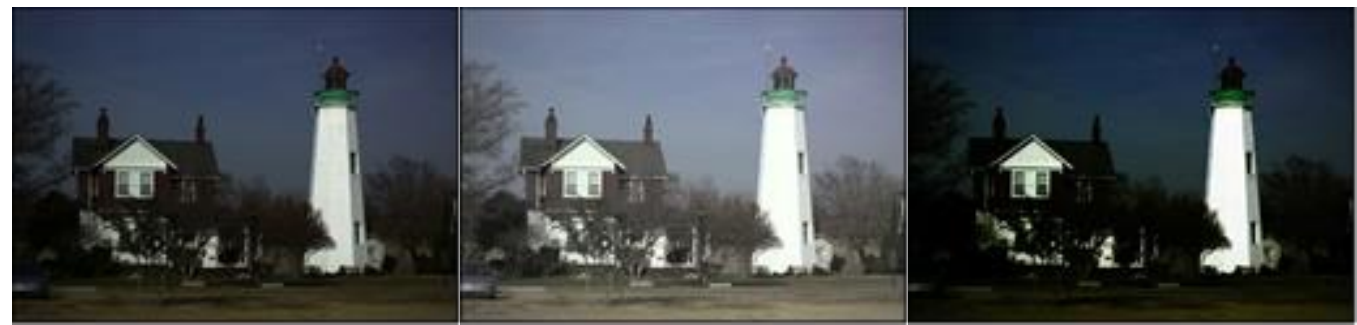

(a)

(b)

(c)

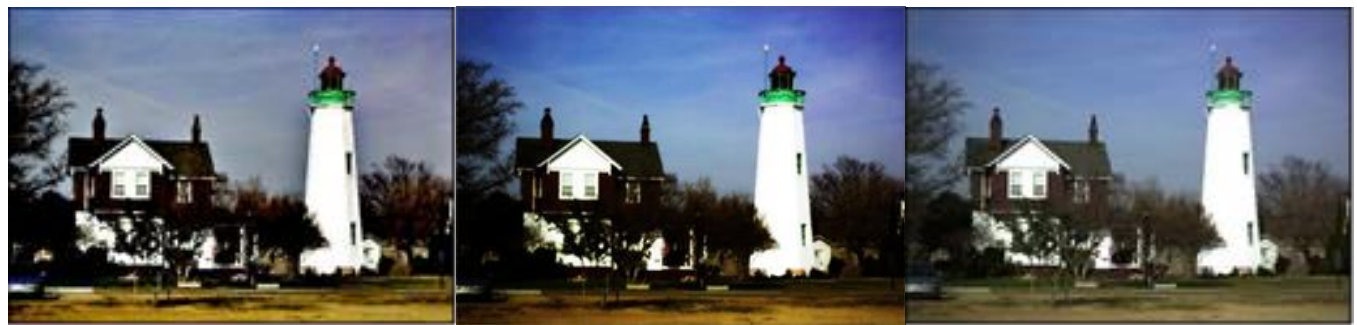

(d)

(e)

(f)

Figure. 5 Contrast improved minaret images in: (a) original, (b) FLBSMF, (c) FLSM, (d) MSRCR, (e) FLBGC, and (f) FLBH

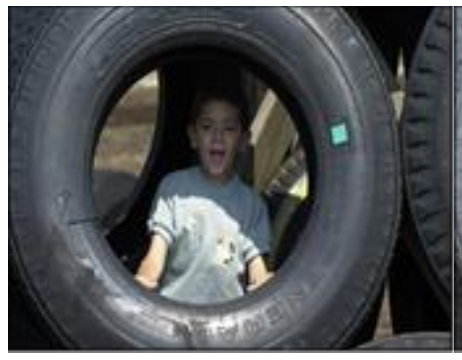

(a)

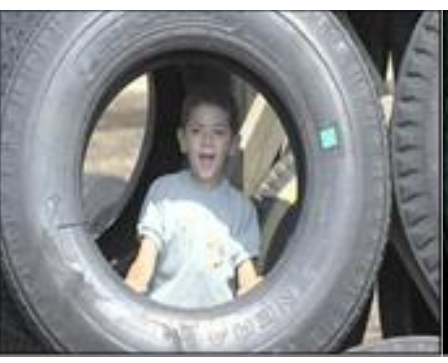

(b)

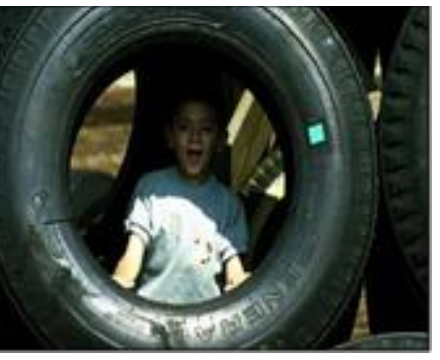

(c)

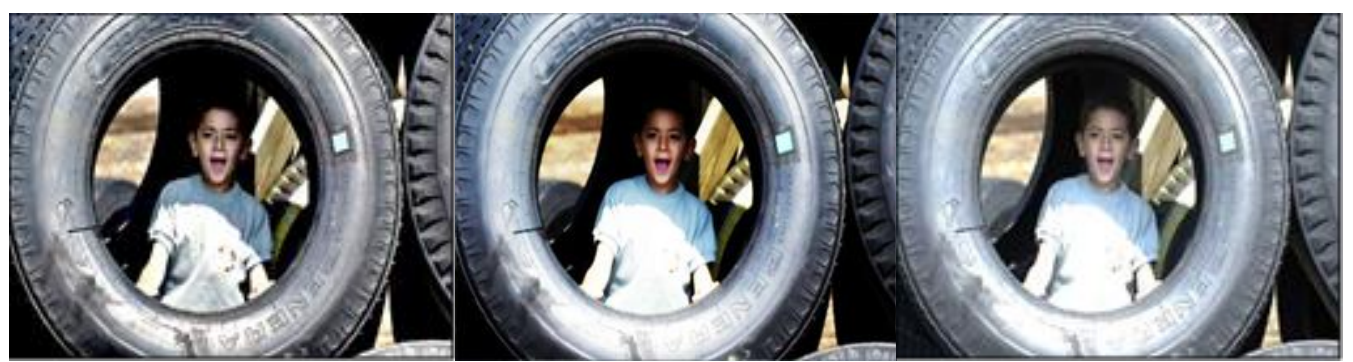

(d)

(e)

(f)

Figure. 6 Contrast improved tire images in: (a) original, (b) FLBSMF, (c) FLSM, (d) MSRCR, (e) FLBGC, and (f) FLBH

with the other algorithms, the proposed method also had the good high value of entropy and lower values for the other four parameters (MSE-H, MSE$S$, NIQE and LOE).

\section{References}

[1] R. Gonzales and R Woods, Digital Image Processing, ed: Prentice hall New Jersey, 2002.

[2] R. Gonzales and R Woods, Digital Image Processing, Addison-Wesley, London, 1977.

[3] H. Daway, F. Mohammed, and D. Abdulabbas, "Aerial image enhancement using modified fast visibility restoration based on sigmoid function",
Advances in Natural and Applied Sciences, Vol. 10, No. 11, pp. 16-22, 2016.

[4] H. Daway and E. Daway, "Underwater Image Enhancement using Colour Restoration based on YCbCr Colour Model", In: Proc. of IOP Conf. Series: Materials Science and Engineering, Vol. 571, No. 1: pp. 1-7, 2019.

[5] Z. AMEER, H. Daway, and H. Kareem, "Enhancement underwater image using histogram equalization based on color restoration", Journal of Engineering and Applied Sciences, Vol. 14, No. 2: pp. 641-647, 2019. 


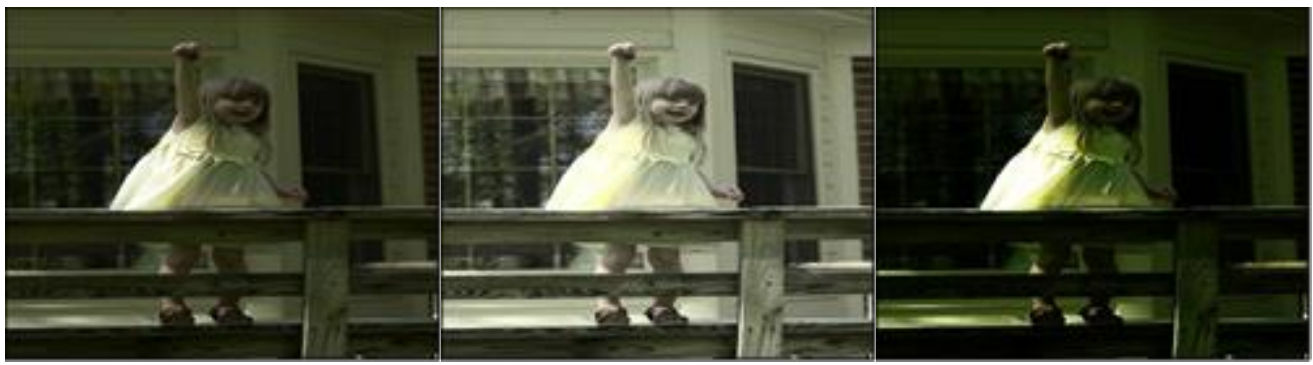

(a)

(b)

(c)

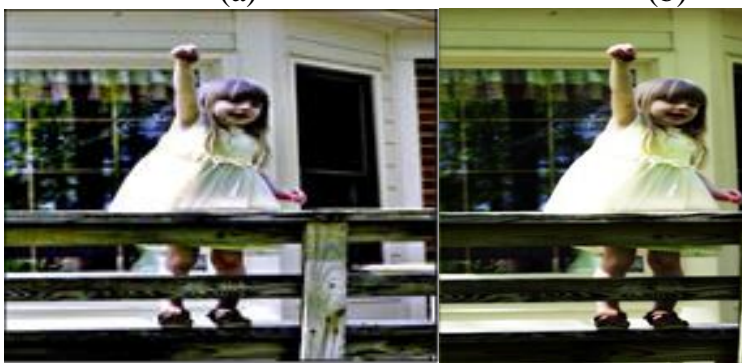

(d)

(e)

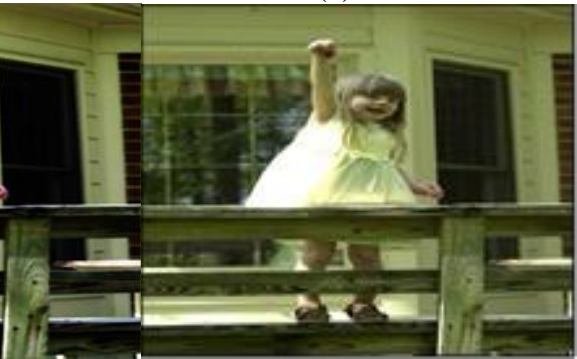

(f)

Figure. 4 Contrast-improved girl_2 images in: (a) original, (b) FLBSMF, (c) FLSM, (d) MSRCR, (e) FLBGC, and (f) FLBH.

[6] N. Mirza, H. Kareem, and H. Daway, "Low lightness enhancement using nonlinear filter based on power function", Journal of Theoretical and Applied Information Technology, Vol.96. No 1, P. 61-70, 2019.

[7] S. Hassan, H. Daway, and I. Al-Alaway, "Improving an Illumination System in the Microscopic Imaging of Nuclear Tracks Using Light Emitting Diode", Indian Journal of Public Health Research \& Development, Vol. 9, NO. 12, pp. 1282-1287, 2018.

[8] Jobson, Z. Rahman, and G. Woodell, "A multiscale retinex for bridging the gap between color images and the human observation of scenes", IEEE Transactions on Image Processing, Vol. 6, No. 7, pp. 965-976, 1997.

[9] L. Zadeh, "Outline of a new approach to the analysis of complex systems and decision processes", IEEE Transactions on Systems, Man, and Cybernetics, No. 1, pp. 28-44, 1973.

[10] L. Zadeh, K. Fu, K. Tanaka, M. Shimura, and C. Negoita, "Fuzzy sets and their applications to cognition and decision processes", IEEE Transactions on Systems, Man, and Cybernetics, Vol. 7, No. 2, pp. 122-123, 1977.

[11] T. Mahashwari and A. Asthana, "Image enhancement using fuzzy technique", International Journal of Research in Engineering Science and Technology, Vol. 2, No. 2, pp. 1-4, 2013.

[12] T. Agarwal, M. Tiwari, and S. Lamba, "Modified histogram based contrast enhancement using homomorphic filtering for medical images", In: Proc. of IEEE International
Advance Computing Conf. (IACC), pp. 964-968, 2014.

[13] S. Chong, K. Sim, and M. Nia, "Modified HL contrast enhancement technique for breast MR images", In: Proc. of IEEE International Conf. on Signal and Image Processing Applications, pp. 360-364, 2013.

[14] Z. Chen, B. Abidi, D. Page, and M. Abidi, "Gray-level grouping (GLG): an automatic method for optimized image contrast Enhancement-part I: the basic method", IEEE Transactions on Image Processing, Vol. 15, No. 8, pp. 2290-2302, 2006.

[15] Z. Chen, B. Abidi, D. L. Page, and M. AAbidi, "Gray-level grouping (GLG): an automatic method for optimized image contrast enhancement-part II: the variations", IEEE Transactions on Image Processing, Vol. 15, No. 8, 2303- 2314, 2006.

[16] M. Hanmandlu and D. Jha, "An optimal fuzzy system for color image enhancement", IEEE Transactions on Image Processing, Vol. 15, No. 10, pp. 2956-2966, 2006.

[17] H. Kundra, E. Aashima, and E. Verma, "Image enhancement based on fuzzy logic", International Journal of Computer Science and Network Security, Vol. 9, No. 10, pp. 141-145, 2009.

[18] B. Jayaram, K. Narayana, and V. Vetrivel, "Fuzzy inference system based contrastenhancement", In: Proc. of the 7th Conf. of the European Society for Fuzzy Logic and Technology: Atlantis Press, pp. 311-318, 2011. 
[19] M. SJ and M. Rajeswari, "Membership Function modification for Image Enhancement using fuzzy logic", International Journal of Emerging Trends \& Technology in Computer Science (IJETTCS), Vol. 2, No. 2, 2013.

[20] G. Raju and M. Nair, "A fast and efficient color image enhancement method based on fuzzylogic and histogram", AEU-International Journal of Electronics and Communications, Vol. 68, No. 3, pp. 237-243, 2014.

[21] Mikhov, Y. Kondratenko, G. Kondratenko, and I. Sidenko, "Fuzzy Logic Approach to Improving the Digital Images Contrast", In: Proc. of the IEEE 2nd Ukraine Conf. on Electrical and Computer Engineering, pp. 11831188, 2019.

[22] D. Zhang, B. Zhan, G. Yang, and X. Hu, "An improved edge detection algorithm based on image fuzzy enhancement", In: Proc. of the 4th IEEE Conf. on Industrial Electronics and Applications, IEEE, pp. 2412-2415, 2009.

[23] S. Wine and R. Horne, The Color Image Processing Hand Book, International Thomson. 1998.

[24] T. Ross, Fuzzy Logic with Engineering Applications, John Wiley \& Sons, 2005.

[25] Zhou, Z. Jia, J. Yang, and N. Kasabov, "Method of improved fuzzy contrast combined adaptive threshold in NSCT for medical image enhancement", BioMed Research International, Vol. 2017, 2017.

[26] S. Wang, J. Zheng, H. Hu, and B. Li, "Naturalness Preserved Enhancement Algorithm for Non-Uniform Illumination Images", IEEE Transactions on Image Processing, Vol. 22 No. 9 pp. 3538-3548. 2013.

[27] A. Mittal, R. Soundararajan, and A. C. Bovik, "Making a "Completely Blind Image Quality Analyzer", IEEE Signal Processing Letters, Vol. 20, No. 3 pp. 209-212, 2013. 